\title{
Influence of Multiple Scattering on Parametric X-Ray Radiation Excited by a Beam of Relativistic Electrons in a Single Crystal
}

\author{
M. V. Alyabyevac, S. V. Blazhevich ${ }^{a, * *}$, A. S. Gorlov ${ }^{b}$, A. V. Noskov ${ }^{a, b}$, , and A. E. Fedoseev ${ }^{a}$ \\ ${ }^{a}$ Belgorod State University, Belgorod, 308015 Russia \\ ${ }^{b}$ Belgorod State Technological University named after V.G. Shukhov, Belgorod, 308012 Russia \\ ${ }^{c}$ Belgorod University of Cooperation, Economics, and Law, Belgorod, 308023 Russia \\ *e-mail:noskovbupk@mail.ru \\ **e-mail:blazh@bsu.edu.ru
}

Received October 20, 2020; revised December 25, 2020; accepted December 30, 2020

\begin{abstract}
Parametric X-ray radiation generated by a beam of relativistic electrons in a single-crystal wafer is studied in the Bragg geometry under conditions of multiple electron scattering at target atoms. Expressions are obtained that describe the spectral-angular and angular radiation density under conditions of multiple electron scattering. An increase in the influence of multiple scattering on the spectral-angular radiation density is demonstrated both with an increase in the target thickness and a decrease in the energy of relativistic electrons. It is shown that the asymmetry of the electron-field reflection relative to the target surface considerably affects the spectral-angular and angular densities of parametric X-ray radiation under the condition of strong multiple scattering.
\end{abstract}

Keywords: single crystal, parametric X-ray radiation, multiple scattering, relativistic electron beam DOI: $10.1134 / \mathrm{S} 1027451021030204$

\section{INTRODUCTION}

Parametric X-ray radiation (PXR) arises due to scattering of the Coulomb field of a relativistic electron by systems of parallel atomic planes of the crystal [1-3]. Currently, there are kinematic [4, 5] and dynamic [2, 3] approaches to the description of PXR. In contrast to the dynamic kinematic approach, it takes into account the interaction of each atom only with the primary or refracted wave in a single crystal, i.e., the interaction of an atom with the wave field, which is created in a single crystal as a result of aggregate scattering at other atoms, is neglected. Significant progress in describing the PXR of relativistic electrons in single crystals has been achieved using the dynamic approach [6, 7]. Clear confirmation of the objectivity and expediency of using the theory of PXR is experimental observation of the peak of parametric X-ray radiation along the velocity of a relativistic electron [8], which is not predicted by kinematic theory.

Further development of the dynamic theory of the parametric X-ray radiation of a relativistic electron in a single crystal in the Laue and Bragg scattering geometry was presented in $[9,10]$ in the general case of asymmetric electron-field reflection with respect to the target surface, when a system of parallel reflecting layers of a target can be located at any given angle to the target surface. The theory of coherent X-ray radiation for beams of relativistic electrons in a single crys- tal in the Laue scattering geometry was developed in [11], where the influence of the divergence of a beam of relativistic electrons on coherent $\mathrm{X}$-ray radiation was shown. In [12], the dynamic theory of the coherent X-ray radiation of a diverging beam of relativistic electrons generated in a single-crystal wafer in the Laue scattering geometry was considered under conditions of the multiple scattering of incident particles. The conditions are shown under which the contribution of diffracted bremsstrahlung radiation can be neglected.

This work is devoted to studying the spectral-angular characteristics of PXR excited by a beam of relativistic electrons crossing a single-crystal wafer in the Bragg scattering geometry, taking into account multiple scattering at target atoms. To take into account multiple scattering, we use the traditional method of averaging the spectral-angular and angular radiation densities over an expanding electron beam with rectilinear trajectories. The influence of multiple scattering and asymmetry of the reflection of the electron field relative to the target surface on the PXR spectralangular density is investigated.

\section{GEOMETRY OF THE RADIATION PROCESS}

Let us consider a beam of relativistic electrons crossing a single crystal in the Bragg-scattering geom- 


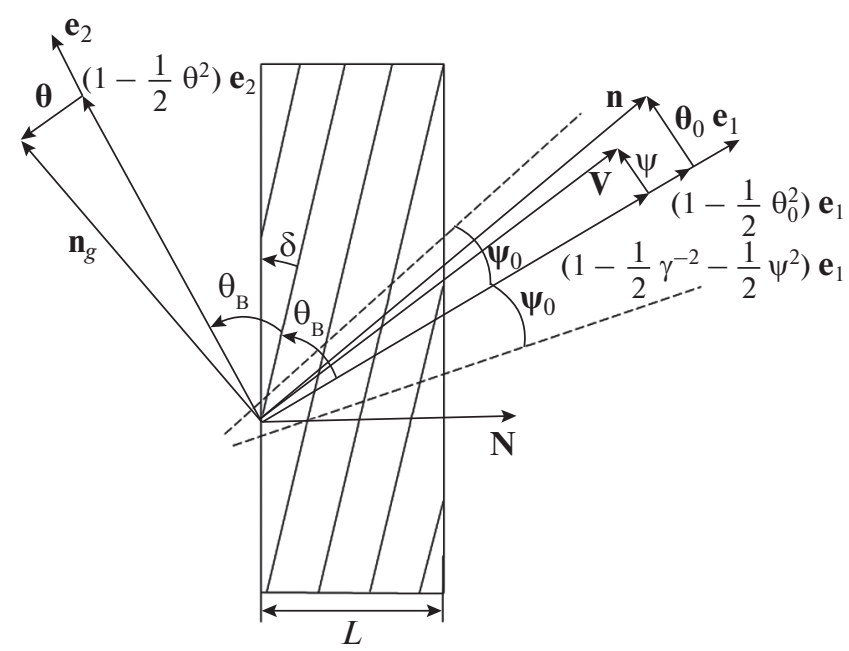

Fig. 1. Geometry of the irradiation process.

etry (Fig. 1). We introduce the angular variables $\boldsymbol{\psi}, \boldsymbol{\theta}$ and $\boldsymbol{\theta}_{0}$ in accordance with the definition of the velocity of a relativistic electron isolated in the beam $\mathbf{V}$ and unit vectors $\mathbf{n}$ in the direction of the photon momentum emitted near the direction of the electron-velocity vector, and $\mathbf{n}_{g}$ in the direction of Bragg scattering:

$$
\begin{gathered}
\mathbf{V}=\left(1-\frac{1}{2} \gamma^{-2}-\frac{1}{2} \psi^{2}\right) \mathbf{e}_{1}+\boldsymbol{\psi}, \quad \mathbf{e}_{1} \boldsymbol{\psi}=0, \\
\mathbf{n}=\left(1-\frac{1}{2} \theta_{0}^{2}\right) \mathbf{e}_{1}+\boldsymbol{\theta}_{0}, \quad \mathbf{e}_{1} \boldsymbol{\theta}_{0}=0, \quad \mathbf{e}_{1} \mathbf{e}_{2}=\cos 2 \theta_{\mathrm{B}}, \\
\mathbf{n}_{g}=\left(1-\frac{1}{2} \theta^{2}\right) \mathbf{e}_{2}+\boldsymbol{\theta}, \quad \mathbf{e}_{2} \boldsymbol{\theta}=0,
\end{gathered}
$$

where $\boldsymbol{\theta}$ is the angle of radiation, measured from the axis of the radiation detector $\mathbf{e}_{2}, \boldsymbol{\psi}$ is the angle of deviation of the considered electron in the beam, measured from the axis of the electron beam $\mathbf{e}_{1}, \boldsymbol{\theta}_{0}$ is the angle between the direction of propagation of the incident photon and the axis $\mathbf{e}_{1}$, and $\gamma=1 / \sqrt{1-V^{2}}$ is the Lorentz factor of the electron. The angular variables are considered as the sum of components parallel and perpendicular to the sectional plane: $\boldsymbol{\theta}=\boldsymbol{\theta}_{\|}+\boldsymbol{\theta}_{\perp}$, $\boldsymbol{\theta}_{0}=\boldsymbol{\theta}_{0 \|}+\boldsymbol{\theta}_{0 \perp}, \boldsymbol{\psi}=\boldsymbol{\Psi}_{\|}+\boldsymbol{\psi}_{\perp}$, and $\psi_{0}$ is the initial divergence of the electron beam.

\section{SPECTRAL-ANGULAR DENSITY}

In [13], the theory of the coherent $\mathrm{X}$-ray radiation of a beam of relativistic electrons in a single crystal in the direction close to the beam axis in the Bragg-scattering $\mathbf{n}$ geometry was developed (Fig. 1). Expressions were obtained that describe the spectral-angular characteristics of parametric X-ray radiation near the velocity of a relativistic electron. With similar reasoning and using similar designations in this work, an expression is obtained for the spectral-angular density of the PXR of a relativistic electron, taking into account the deviation of the direction of its velocity $\mathbf{V}$ relative to the electron-beam axis $\mathbf{e}_{1}$ (angle $\psi\left(\psi_{\perp}, \psi_{\|}\right)$):

$$
\begin{gathered}
\omega \frac{d^{2} N_{\mathrm{PXR}}^{(s)}}{d \omega d \Omega}=\frac{e^{2}}{\pi^{2}} \frac{\Omega^{(s) 2}}{\left(\Delta-\chi_{0}^{\prime}\right)^{2}} R_{\mathrm{PXR}}^{(s)}, \\
R_{\mathrm{PXR}}^{(s)}=\frac{\left(\xi^{(s)}+\sqrt{\xi^{(s) 2}-\varepsilon}\right)^{2}}{\xi^{(s) 2}-\varepsilon+\varepsilon \sin ^{2}\left(\frac{b^{(s)} \sqrt{\xi^{(s) 2}-\varepsilon}}{\varepsilon}\right)} \\
\times \frac{\sin ^{2}\left(\frac{b^{(s)}}{2}\left(\frac{\xi^{(s)}+\sqrt{\xi^{(s) 2}-\varepsilon}}{\varepsilon}-\sigma^{(s)}\right)\right)}{\left(\frac{\xi^{(s)}+\sqrt{\xi^{(s) 2}-\varepsilon}}{\varepsilon}-\sigma^{(s)}\right)^{2}},
\end{gathered}
$$

where

$$
\begin{gathered}
\sigma^{(s)}=\frac{1}{\left|\chi_{g}^{\prime}\right| C^{(s)}}\left(\gamma^{-2}+\left(\theta_{\perp}-\psi_{\perp}\right)^{2}+\left(\theta_{\|}+\psi_{\|}\right)^{2}-\chi_{0}^{\prime}\right), \\
\xi^{(s)}(\omega)=\eta^{(s)}(\omega)+\frac{1+\varepsilon}{2 v^{(s)}}, \quad \eta^{(s)}(\omega)=\frac{2 \sin ^{2} \theta_{\mathrm{B}}}{V^{2}\left|\chi_{g}^{\prime}\right| C^{(s)}} \\
\times\left(1-\frac{\omega\left(1-\theta_{\|} \operatorname{ctg} \theta_{\mathrm{B}}\right)}{\omega_{\mathrm{B}}}\right), \quad v^{(s)}=\frac{\chi_{g}^{\prime} C^{(s)}}{\chi_{0}^{\prime}}, \\
\varepsilon=\frac{\left|\gamma_{g}\right|}{\left|\gamma_{0}\right|}=\frac{\sin \left(\theta_{\mathrm{B}}-\delta\right)}{\sin \left(\theta_{\mathrm{B}}+\delta\right)}, \quad \Delta\left(\theta_{\perp}, \theta_{\|}, \psi_{\perp}, \psi_{/ /}\right) \\
=\gamma^{-2}+\left(\theta_{\perp}-\psi_{\perp}\right)^{2}+\left(\theta_{\|}+\psi_{\|}\right)^{2}, \\
b^{(s)}=\frac{1}{2 \sin \left(\theta_{\mathrm{B}}+\delta\right)} \frac{L}{L_{\mathrm{ext}}^{(s)}}, \quad L_{\mathrm{ext}}^{(s)}=1 / \omega\left|\chi_{g}^{\prime}\right| C^{(s)} .
\end{gathered}
$$

At a fixed value of $\theta_{B}$, the asymmetry parameter $\varepsilon$ defines the orientation of the input target surface relative to the reflecting layers, which is determined by the angle $\delta$. Parameter $b^{(s)}$ is equal to half the path of the electron in the target $L_{e}=L / \sin \left(\theta_{\mathrm{B}}+\delta\right)$, which is expressed in $\mathrm{X}$-ray extinction lengths in the crystal $L_{\text {ext }}^{(s)}=1 / \omega\left|\chi_{g}^{\prime}\right| C^{(s)}$.

The angular density of the PXR is obtained from (1) after integration over the frequency function $\xi^{(s)}(\omega)$, using the ratio $\frac{d \omega}{\omega}=-\frac{\left|\chi_{\mathrm{g}}^{\prime}\right| C^{(s)}}{2 \sin ^{2} \theta_{\mathrm{B}}} d \xi^{(s)}$, which follows from the expression for $\xi^{(s)}(\omega)$ in 2):

$$
\begin{aligned}
& \frac{d N_{\mathrm{PXR}}^{(s)}}{d \Omega}=\frac{e^{2}}{\pi^{2}} \frac{\left|\chi_{g}^{\prime}\right| C^{(s)}}{2 \sin ^{2} \theta_{\mathrm{B}}} \frac{\Omega^{(s) 2}}{\left(\Delta-\chi_{0}^{\prime}\right)^{2}} \int_{\sqrt{\varepsilon}}^{\infty} R_{\mathrm{PXR}}^{(s)} d \xi^{(s)} . \\
& @[\text { Кеу: ПРИ } \rightarrow \text { PXR] }
\end{aligned}
$$


Since the PXR spectrum is very narrow under the condition $b^{(s)} \gg 1$, for integration, we use the wellknown approximation $\frac{\sin ^{2}(a x)}{x^{2}} \rightarrow \pi a \delta(x)$ and obtain the angular density of the PXR:

$$
\begin{gathered}
\frac{d N_{\mathrm{PXR}}^{(s)}}{d \Omega}=\frac{e^{2}}{2 \pi \sin ^{2} \theta_{\mathrm{B}}\left|\chi_{g}^{\prime}\right| C^{(s)}} \\
\times \Omega^{(s)^{2}} \varepsilon^{2} \frac{\sigma^{(s)^{2}} \varepsilon-1}{\left(\sigma^{(s)^{2}} \varepsilon-1\right)^{2}+2 \varepsilon \sigma^{(s)^{2}}} b^{(s)} .
\end{gathered}
$$

Since electrons are multiply scattered at atoms of the medium, we will average the spectral-angular and angular PXR density over the angular distribution of electrons in the beam in the form of a Gaussian function, which varies with the path length in the target $t$ due to the multiple scattering of an electron:

$$
f(\psi, t)=\frac{1}{\pi\left(\psi_{0}^{2}+\psi_{s}^{2} t\right)} \exp \left(-\frac{\psi^{2}}{\psi_{0}^{2}+\psi_{s}^{2} t}\right),
$$

i.e., averaging over an expanding beam of emitting electrons with rectilinear trajectories along the path length of an electron in the target $L_{e}=L / \sin \left(\theta_{\mathrm{B}}+\delta\right)$, where $\psi_{0}$ is the initial divergence of the electron beam, $\psi_{s}^{2}=\frac{E_{s}^{2}}{m^{2} \gamma^{2}} \frac{1}{L_{R}}\left(1+0.038 \ln \left(\frac{t}{L_{R}}\right)\right)^{2}$ is the mean square of the angle of multiple scattering of an electron per unit length, taking into account its dependence on the length of the path traveled $t$ in a single crystal [14], $E_{s}$ $\approx 21 \mathrm{MeV}$. The averaged expressions for the spectralangular and angular PXR density taking into account multiple scattering take the form:

$$
\begin{aligned}
&\left\langle\omega \frac{d^{2} N_{\mathrm{PXR}}^{(s)}}{d \omega d \Omega}\right\rangle=\frac{e^{2}}{\pi^{2}} \frac{1}{L_{e}} \int_{0}^{L_{e}} \int_{-\infty}^{\infty} \int_{-\infty}^{\infty} R_{\mathrm{PXR}}^{(s)} \frac{\Omega^{(s)^{2}}\left(\psi_{\perp}, \psi_{\|}\right)}{\left(\Delta\left(\psi_{\perp}, \psi_{\|}\right)-\chi_{0}^{\prime}\right)^{2}} \\
& \times \frac{\exp \left(-\frac{\psi^{2}}{\psi_{0}^{2}+\psi_{s}^{2} t}\right)}{\pi\left(\psi_{0}^{2}+\psi_{s}^{2} t\right)} d \psi_{\perp} d \psi_{\|} d t \\
&\left\langle\frac{d N_{\mathrm{PXR}}^{(s)}}{d \Omega}\right\rangle=\frac{1}{L_{e}} \int_{0}^{L_{e}} \int_{-\infty}^{\infty} \int_{-\infty}^{\infty} \frac{d N_{\mathrm{PXR}}^{(s)}}{d \Omega} \\
& \times \frac{\exp \left(-\frac{\psi^{2}}{\psi_{0}^{2}+\psi_{s}^{2} t}\right)}{\pi\left(\psi_{0}^{2}+\psi_{s}^{2} t\right)} d \psi_{\perp} d \psi d t_{\|} .
\end{aligned}
$$

In the absence of multiple scattering, we will average the spectral-angular and angular PXR densities over all possible rectilinear electron trajectories in the beam according to the Gaussian function $f(\psi, t)=\frac{1}{\pi \psi_{0}^{2}} \exp \left(-\frac{\psi_{\perp}^{2}+\psi_{0}^{2}}{\psi_{0}^{2}}\right)$. In this case, we will leave the designations unchanged:

$$
\begin{aligned}
\omega \frac{d^{2} N_{\mathrm{PXR}}^{(s)}}{d \omega d \Omega} & =\int_{-\infty}^{\infty} \int_{-\infty}^{\infty} d \psi_{\perp} d \psi_{\|} \frac{\exp \left(-\frac{\psi^{2}}{\psi_{0}^{2}}\right)}{\pi \psi_{0}^{2}} \omega \frac{d^{2} N_{\mathrm{PXR}}^{(s)}}{d \omega q \Omega}, \\
\frac{d N_{\mathrm{PXR}}^{(s)}}{d \Omega} & =\int_{-\infty}^{\infty} \int_{-\infty}^{\infty} d \psi_{\perp} d \psi_{\|} \frac{\exp \left(-\frac{\psi^{2}}{\psi_{0}^{2}}\right)}{\pi \psi_{0}^{2}} \frac{d N_{\mathrm{PXR}}^{(s)}}{d \Omega} .
\end{aligned}
$$

\section{ANALYSIS OF THE SPECTRAL-ANGULAR DENSITY OF PXR: NUMERICAL CALCULATIONS}

We consider a beam of relativistic electrons with the energy $E=m \gamma$, crossing a single-crystal C(111) carbon wafer with the thickness $L$ (Fig. 1). Let the axis of the beam of relativistic electrons $\mathbf{e}_{1}$ be located at an angle of $\theta_{\mathrm{B}}=16.2^{\circ}$ to the considered reflecting system of parallel atomic planes of the single crystal; the Bragg frequency is $\omega_{B}=10.9 \mathrm{keV}$. Numerical calculations will be carried out for $\sigma$-polarized waves $(s=1)$.

Let us consider the effect of multiple scattering on the spectral-angular density of PXR with a fixed observation angle $\theta$. Figure 2 shows curves constructed by formulas (6a) and (7a), describing the spectral-angular densities of PXR for a fixed observation angle of $\theta_{\perp}=\sqrt{\gamma^{-2}-\chi_{0}^{\prime}} \approx 4.84 \mathrm{mrad}, \theta_{\perp}=0$ at the maximum angular density of PXR at a target thickness

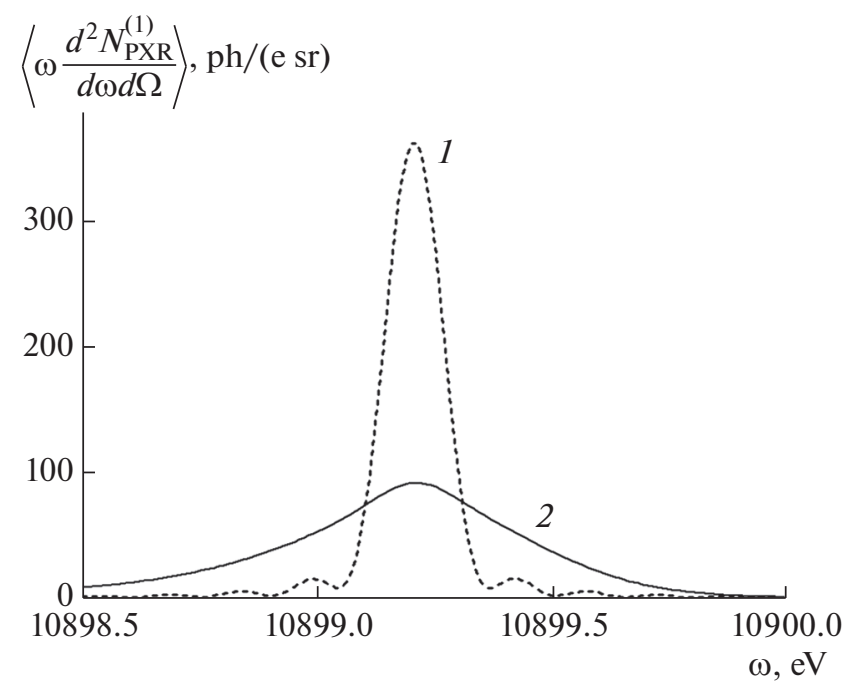

Fig. 2. PXR spectral-angular densities at a fixed observation angle $\boldsymbol{\theta}\left(\theta_{\perp}, \theta_{\|}\right)$excluding ( 1$)$ and taking into account (2) multiple scattering: $L=15 \mu \mathrm{m}, \varepsilon=1, \gamma=300, \theta_{\perp}=$ $4.84 \mathrm{mrad}, \theta_{\|}=0, \psi_{0}=1 \mathrm{mrad}$. Here and below, ph is a photon and $\mathrm{e}$ is an electron. 


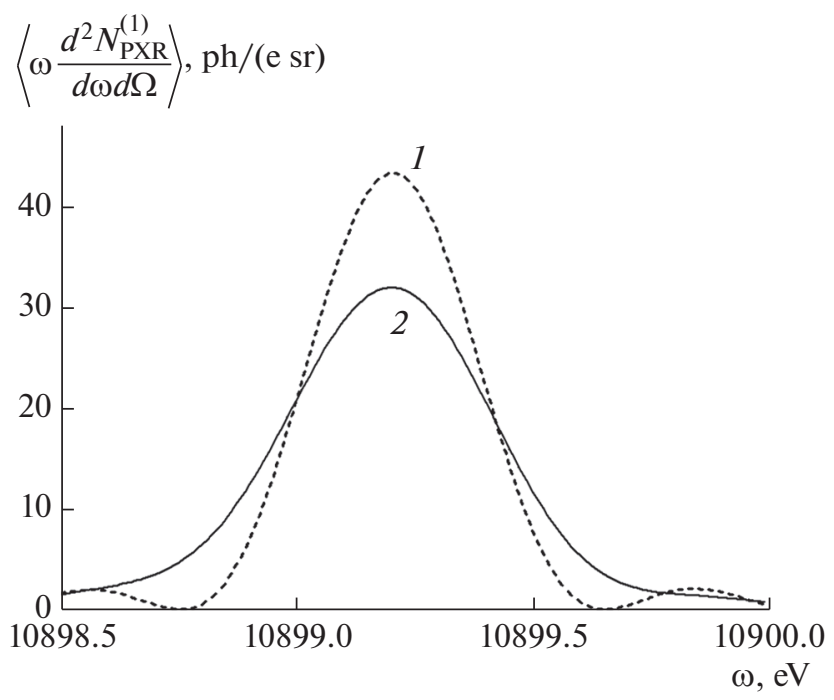

Fig. 3. Same as in Fig. 2, but at $L=5 \mu \mathrm{m}$.

of $L=15 \mu \mathrm{m}$. The dotted line corresponds to PXR without taking into account multiple scattering and the solid line, taking into account multiple scattering. It can be seen that, under the conditions under consideration, the multiple scattering of beam electrons significantly affects the spectral-angular PXR density. Figure 3 shows curves similar to Fig. 2, but with a smaller target thickness of $L=5 \mu \mathrm{m}$. It can be seen that with a decrease in the target thickness, the effect of multiple scattering is significantly weakened, which is associated with a decrease in the path of the electron in the target. Figure 4 shows curves similar to Fig. 3, but at a three times lower electron energy $(\gamma=100)$. Figure 4 implies the significant effect of the multiple scattering of relativistic electrons by atoms of the medium, which is a consequence of an increase in the mean square of the angle of multiple scattering with decreasing energy.

Let us consider the angular PXR density and the effect of multiple PXR scattering on it. Figure 5 shows the curves plotted by formulas (6b) and (7b) describing the angular densities of PXR for different target thicknesses $L$. The dashed curve corresponds to the angular density of PXR without taking into account multiple scattering, and the solid curve, taking into account multiple scattering. Figure 5 shows an increase in the PXR angular density with increasing target thickness. It is seen that under the conditions under consideration at $L=10 \mu \mathrm{m}$ and above, the multiple scattering of beam electrons significantly affects the PXR angular density.

Let us consider the influence of the asymmetry of the reflection of the electron field relative to the target surface on the spectral-angular and angular PXR densities under conditions of the multiple scattering of relativistic electrons at target atoms. The reflection asymmetry is determined by the parameter

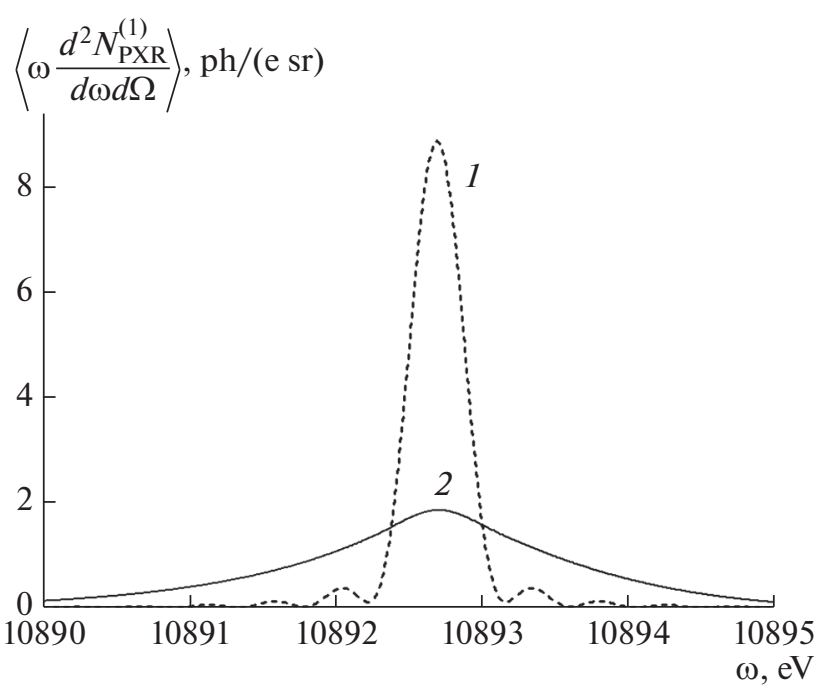

Fig. 4. PXR spectral-angular densities at a fixed observation angle $\boldsymbol{\theta}\left(\theta_{\perp}, \theta_{\|}\right)$excluding (1) and taking into account (2) multiple scattering: $L=15 \mu \mathrm{m}, \varepsilon=1, \gamma=100, \theta_{\perp}=11 \mathrm{mrad}$, $\theta_{\|}=0, \psi_{0}=1 \mathrm{mrad}$.

$\varepsilon=\frac{\sin \left(\theta_{\mathrm{B}}-\delta\right)}{\sin \left(\theta_{\mathrm{B}}+\delta\right)}$. By changing the angle $\delta$ between the target surface and the system of parallel atomic planes of the single crystal (Fig. 1), we change the parameter $\varepsilon$ at the fixed Bragg angle $\theta_{\mathrm{B}}$. In the case when $\varepsilon>1$, the angle $\delta<0$, Fig. 1 shows a positive angle $\delta$. Figure 6 shows the curves plotted by formula (6a), describing the spectral-angular density of PXR under conditions of strong multiple scattering (similar to the curves in Fig. 2), for various asymmetry parameters $\varepsilon: \varepsilon=1(\delta=0)$,

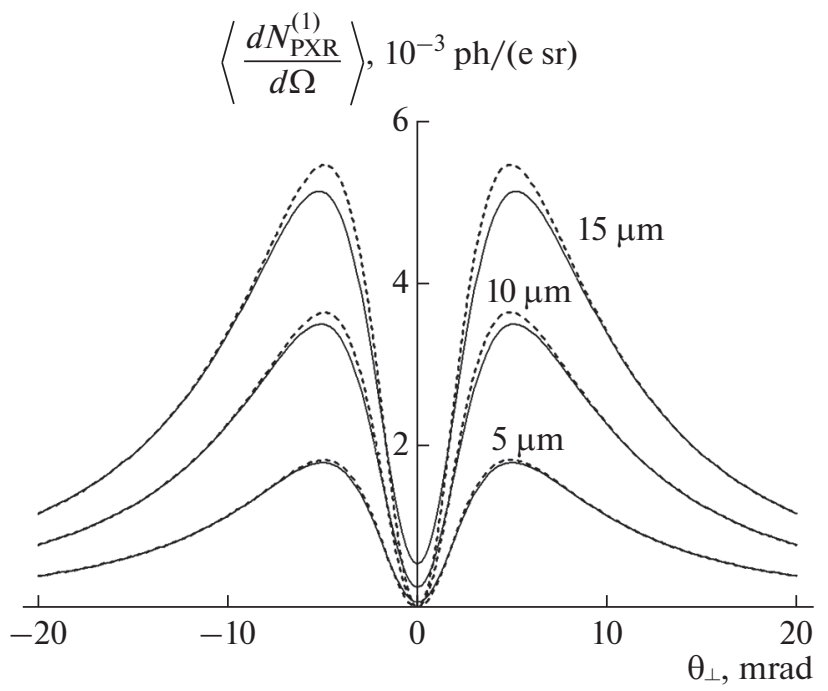

Fig. 5. PXR angular densities without (dotted lines) and with (solid lines) multiple scattering for different target thicknesses $L: \varepsilon=1, \gamma=300, \psi_{0}=1 \mathrm{mrad}$. 


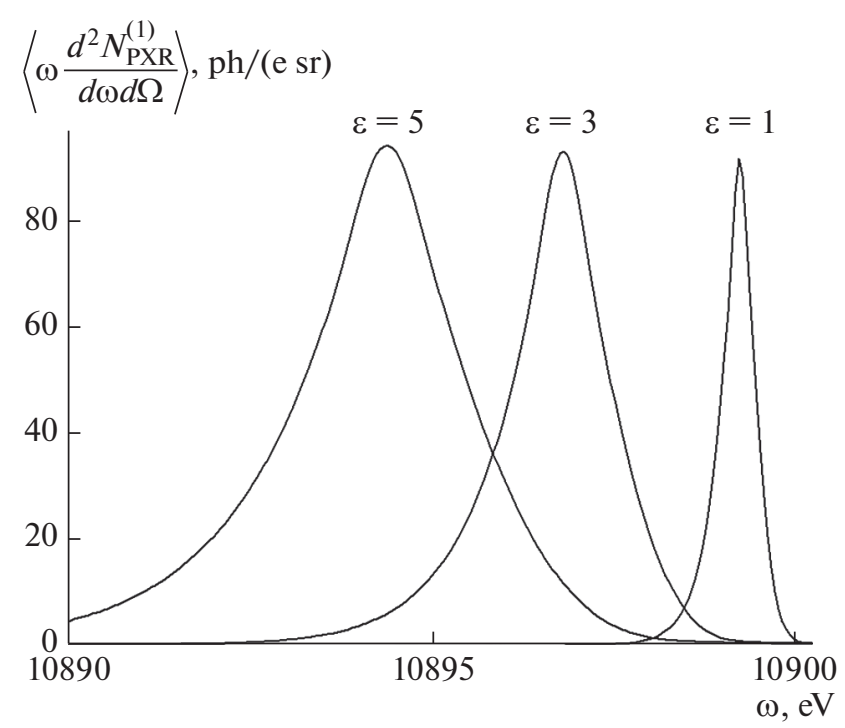

Fig. 6. PXR spectral-angular densities for various asymmetry parameters $\varepsilon: L=15 \mu \mathrm{m}, \gamma=300, \theta_{\perp}=4.84 \mathrm{mrad}$, $\theta_{\|}=0, \psi_{0}=1 \mathrm{mrad}$.

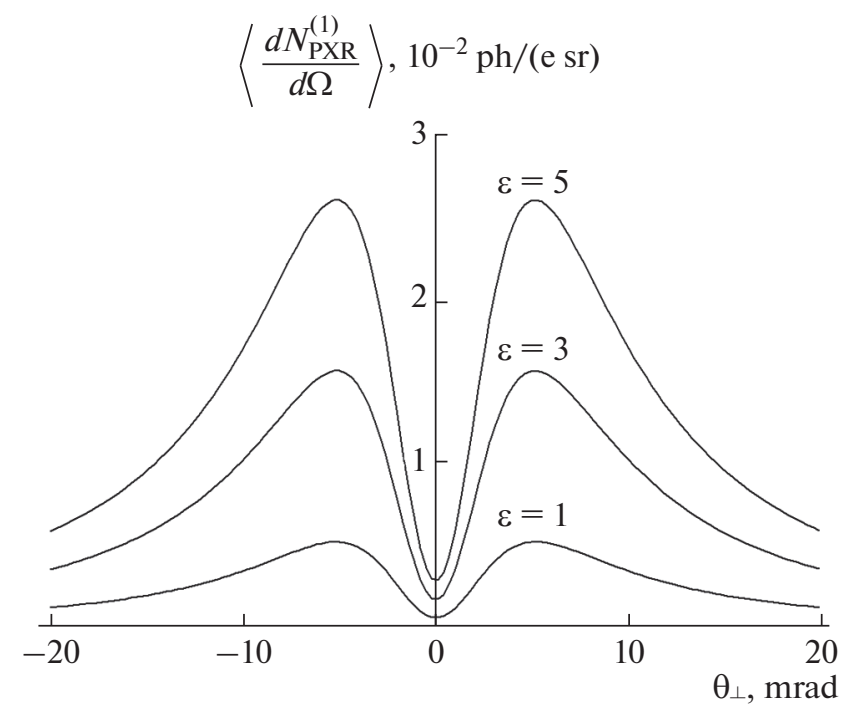

Fig. 7. PXR angular densities for various asymmetry parameters $\varepsilon: L=15 \mu \mathrm{m}, \gamma=300, \psi_{0}=1 \mathrm{mrad}$.

$\varepsilon=3\left(\delta=-8.3^{\circ}\right), \varepsilon=5\left(\delta=-10.9^{\circ}\right)$. Figure 6 shows the significant influence of the asymmetry of reflection on the width of the peak of the PXR spectrum, which can also lead to an increase in the angular density of the PXR (Fig. 7).

\section{CONCLUSIONS}

A dynamic theory of parametric $\mathrm{X}$-ray radiation generated by a beam of relativistic electrons in a single- crystal wafer in the Bragg-scattering geometry under conditions of the multiple scattering of incident particles is developed. Expressions are obtained that describe the spectral-angular and angular PXR densities with and without allowance for multiple scattering of the beam electrons at target atoms. The effect of multiple scattering on the spectral-angular and angular PXR densities is investigated for different target thicknesses and different energies of relativistic electrons. Enhancement of the effect of multiple scattering on the spectral-angular density of PXR is demonstrated with an increase in the target thickness and a decrease in the energy of relativistic electrons. It is shown that asymmetry with respect to the target surface of reflection of the electron field on the spectralangular and angular PXR densities is shown under conditions of the strong multiple scattering of relativistic electrons at target atoms. The results of this work can be useful in setting up new experiments to study the properties of PXR and provide more accurate interpretation of experiments under conditions of dynamic diffraction and reflection asymmetry.

\section{REFERENCES}

1. M. L. Ter-Mikaelyan, Influence of the Environment on Electromagnetic Processes at High Energies (Akad. Nauk ArmSSR, Erevan, 1969) [in Russian].

2. G. M. Garibyan and Yan Shi, Zh. Eksp. Teor. Fiz. 61, 930 (1971).

3. V. G. Baryshevskii, I. D. Feranchuk, Zh. Eksp. Teor. Fiz. 61, 944 (1971).

4. H. Nitta, Phys. Lett. A 158, 270 (1991).

5. I. D. Feranchuk and A. V. Ivashin, J. Phys. 46, 1981 (1985).

6. A. S. Kubankin, N. N. Nasonov, V. I. Sergienko, and I. E. Vnukov, Nucl. Instrum. Methods Phys. Res., Sect. B 201, 97 (2003).

7. N. Nasonov and A. Noskov, Nucl. Instrum. Methods Phys. Res., Sect. B 201, 67 (2003).

8. A. N. Aleinik, A. N. Baldin, E. A. Bogomazova, et al., JETP Lett. 80, 393 (2004).

9. S. V. Blazhevich and A. V. Noskov, Nucl. Instrum. Methods Phys. Res., Sect. B 266, 3770 (2008).

10. S. V. Blazhevich and A. V. Noskov, J. Surf. Invest.: X-Ray, Synchrotron Neutron Tech. 4, 303 (2010).

11. S. V. Blazhevich, G. A. Grazhdankin, R. A. Zagorodnyuk, and A. V. Noskov, Nucl. Instrum. Methods Phys. Res., Sect. B 355, 170 (2015).

12. S. V. Blazhevich, N. I. Moskalenko, T. V. Kos'kova, E. A. Tkachenko, A. V. Noskov, J. Surf. Invest.: X-Ray, Synchrotron Neutron Tech. 11, 49 (2017).

13. S. V. Blazhevich, K. S. Lyushina, A. V. Noskov, J. Exp. Theor. Phys. 128, 212 (2019).

14. R. M. Barnett, C. D. Carone, D. E. Groom, et al., Phys. Rev. D: Part. Fields 54, 1 (1996). 